\title{
Research Paper: Prediction of Body Center of Mass Acceleration From Trunk and Lower Limb Joints Accelerations During Quiet Standing
}

\author{
Leila Ghazaleh $^{1^{*}}$, Mehrdad Anbarian ${ }^{2}$, Mohsen Damavandi ${ }^{3}$
}

1. Department of Sports Physiology, Faculty of Physical Education and Sport Sciences, Alzahra University, Tehran, Iran.

2. Department of Sports Biomechanics, Faculty of Sport Sciences, Bu-Ali Sina University, Hamedan, Iran.

3. Department of Sports Physiology, Faculty of Sport Sciences, Hakim Sabzevari University, Sabzevar, Iran.

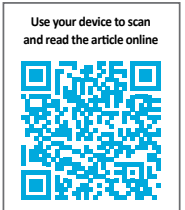

Citation: Ghazaleh L, Anbarian M, Damavandi M. Prediction of Body Center of Mass Acceleration From Trunk and Lower Limb Joints Accelerations During Quiet Standing. Physical Treatments. 2017; 7(2):103-112. http://dx.doi.org/10.32598/ptj.7.2.103

http://dx.doi.org/10.32598/ptj.7.2.103

Article info:

Received: 01 Dec 2016

Accepted: 29 Mar 2017
Keywords:

Balance control, Quiet standing, Joints acceleration, Center of mass, Dominant and nondominant lower limb

\begin{abstract}
A B S T RA C T
Purpose: Predicting body Center of Mass (COM) acceleration is carried out with more accuracy based on the acceleration of three joints of lower limb compared to only accounting joints of hip and ankle. Given that trunk movement during quite standing is noticeable, calculating trunk acceleration in model might increase prediction accuracy of COM acceleration. Moreover, in previous research studies, dominant and nondominant limb was neglected as influencing variables in prediction accuracy. Therefore, this study aimed to investigate the accuracy of predicting COM acceleration based on the accelerations of lower limb joints and trunk, with emphasis on weight distribution on legs.
\end{abstract}

Methods: The relevant kinematic data were collected using motion analysis systems. In this regard, visual 3D software was used to create a 14-segment model for each subject and estimate the positions of body COM. A force plate was used to assess the body weight distribution between legs. Calculation of the summation of joints angular accelerations was done using algebraic addition of time series data.

Results: Prediction of COM acceleration, based on the individual acceleration of lower limb joints and trunk, revealed that just acceleration of hip joint in both dominant and nondominant lower limbs was an appropriate variable for predicting COM acceleration $\left(\mathrm{R}^{2}=0.40\right)$. However, during prediction based on the summation of trunk and joints accelerations, its accuracy showed a significant increase $\left(\mathrm{R}_{\text {adj }}^{2}=0.90\right)$.

Conclusion: The summation of angular accelerations of trunk and lower limb joints is the most accurate predictor of $\mathrm{COM}$ acceleration during quiet standing balance control. Simultaneous changes of lower limb joints and trunk accelerations control COM acceleration.

\footnotetext{
* Corresponding Author:

Leila Ghazaleh, PhD

Address: Department of Sports Physiology, Faculty of Physical Education and Sport Sciences, Alzahra University, Tehran, Iran.

Phone: +98 (21) 85692668

E-mail:l.ghazaleh@alzahra.ac.ir
} 


\section{Introduction}

$\mathrm{T}$

he stabilization of the spatial position of the body Center of Mass (COM) is often assumed to be the goal of postural responses $[1,2]$. Therefore, researchers and clinicians focus on the behavior of body's COM during balance assessment. However, since the measurement of COM variations are technically complicated, cumbersome, costly, and error prone [3], researchers try to develop simple but yet precise methods for assessing the body balance [4-7].

The most common method based on Single-link Inverted Pendulum (SIP) model has been adopted in numerous studies [8-10]. According to SIP model, during quiet standing position, body balance is controlled by the moment of the ankle and body sways as a rigid structure above the ankles [9]. Recent studies, however, have indicated that in addition to ankle joint, other joints play important and significant role in balance control. For example, Günther et al. [11] demonstrated that all leg joints actively contribute in quiet human stance, even in the undisturbed cases. According to the uncontrolled manifold approach, Hsu et al. [12] reported that all body joints are involved in the stabilization of the COM. Yamamoto et al. [13] demonstrated that besides ankle and hip, knee joint motion has substantial effect on the COM kinematics, even during quiet standing. Zhang et al. [14] developed a simplified single-segment, sliding mode control model to track kinematics and kinetics during upright stance. Based on the results of their study, these researchers suggested that subsequent models designed for this purpose should be multi-segment models. Therefore, a more sophisticated approach than SIP model, which takes into account the movements of all lower extremity joints and even trunk, should be applied to assess the balance control.

In order to attain such an approach, some concerns should still be clarified; 1) Do all joints act simultaneously and in coordination during balance control?; 2) How this coordination is observed and measured?; and 3) How does the coordinated performance of joints lead to $\mathrm{COM}$ control?

The answers to these questions help in removing the ambiguities on motor mechanisms and provides a more appropriate criterion for assessing balance based on multi-link pendulum. Some researchers assessed the angular displacement, velocity, and acceleration around the ankle and hip joints during quiet standing balance control $[15,16]$. Their studies demonstrated that coordinated movements of the joints during balance control were associated with their angular accelerations. According to Aramaki et al. [15], a consistent reciprocal relationship exists between the angular accelerations of the hip and ankle joints, while the angular motions around these joints are not supposed to keep COM at a constant position, but rather to minimize its acceleration. Some studies in this field predict $\mathrm{COM}$ acceleration based on the accelerations of lower extremity joints $[13,16]$. Accordingly, Yamamoto [13] demonstrated that to predict more accurately acceleration of COM, knee joint acceleration should be taken into account along with those of the hip and ankle.

Despite these findings, the other related variables should be investigated, since in all previous studies two-dimensional calculations of joints angles were considered. Furthermore, in these studies the movement of all three lower limb joints were investigated, and while the body was assumed to be bilaterally symmetric, the joints of the left leg was studied, without considering the dominant- and non-dominant leg and or manner of weight distribution between them. In addition, aforesaid studies did not assess the coordinated movement of the trunk segment along with the leg joints, even though the movement of trunk was considerable at the quiet standing $[12,17]$. Therefore, to more accurately investigate the coordinated performance of joints during quiet standing balance control, the present study was carried out. It aimed to investigate the prediction of COM acceleration based on joints accelerations of the lower limbs and trunk, with emphasis on the weight distribution on legs.

\section{Materials and Methods}

\section{Participants}

Ten healthy young females (Mean [SD] height: 168.5 [4.9] cm, Mean [SD] weight: 61.3 [5.0] kg, Mean [SD] age: 26.9 [2.2] y) having no history of neurological or musculoskeletal disorders volunteered for the study (Table 1). Before entering the study, the participants signed a consent form approved by the Ethics Committee of Hamedan University of Medical Sciences.

\section{Experiments and procedures}

Study participants stood barefoot in quiet standing position on a force platform, with their feet apart at shoulder width distance and their arms hanging alongside the body. Tests were conducted with open eyes, with participants looking at a plate in front of their eyes, $2.5 \mathrm{~m}$ away. They were asked to stand as motionless as possi- 
Table 1. Characteristics of the subjects

\begin{tabular}{|c|c|c|c|c|}
\hline Subject & Age (y) & Height $(\mathrm{cm})$ & Mass (kg) & BMI $\left(\mathrm{kg} / \mathrm{m}^{2}\right)$ \\
\hline 1 & 25.8 & 162.5 & 60.2 & 22.9 \\
\hline 2 & 29.3 & 167.0 & 72.1 & 25.8 \\
\hline 3 & 29.2 & 175.0 & 59.5 & 19.4 \\
\hline 4 & 29.8 & 168.0 & 61.4 & 21.7 \\
\hline 5 & 24.2 & 174.0 & 64.0 & 21.1 \\
\hline 6 & 24.6 & 165.0 & 64.3 & 23.6 \\
\hline 7 & 25.6 & 163.0 & 52.8 & 19.9 \\
\hline 8 & 24.7 & 167.5 & 57.5 & 20.6 \\
\hline 9 & 27.7 & 176.0 & 61.5 & 19.8 \\
\hline 10 & 28.5 & 167.0 & 60.0 & 21.5 \\
\hline Mean $\pm S D$ & $26.9 \pm 2.2$ & $168.5 \pm 4.9$ & $61.3 \pm 5.0$ & $21.6 \pm 2.0$ \\
\hline
\end{tabular}

PHYSICAL TREA $\mid$ MENTS

ble. Four trials of $60 \mathrm{~s}$ were conducted, with a rest period between the trials.

Fifty six reflective markers (14-mm diameter) were placed over anatomical landmarks (Figure 1). Video data were collected using a video-based Qualysis motion capture camera (Oqus cameras, Sweden) at 200 Hz. Visual 3D software (V4.96.13) was used to create a 14-segment model (hands, forearms, upper arms, trunk, pelvis, thighs, shanks and feet) for each subject [18]. The three-dimensional (3D) kinematic data were used to estimate the 3D positions of body COM and calculate the angular displacements and accelerations of the ankle, knee and hip joints, along with those of the trunk [19]. The hip, knee, and ankle angles were calculated using the relative angles between trunk and thigh, thigh and shank, and shank and foot segments, respectively. The COM acceleration was calculated using MATLAB (v2009b, The Mathworks Inc., Natick, MA, USA). First, COM displacement was calculated, then, its numerical differentiation was performed to calculate the related accelerations. The segmental masses were determined from the total body mass and based on Dempster regression equations [19]. The results were reported only for the anteroposterior direction.

Dominant leg was defined using ball-kick and balance recovery tests [20]. Studies have indicated that humans generally use the nondominant leg for postural stabilization $[21,22]$. Therefore, in the present study both lower limbs were assessed and the opposite limb of the domi- nant leg was considered as the stability or nondominant lower limb during quiet standing [20].

To assess the body weight distribution between the dominant and nondominant lower limbs, force plate data were recorded at $200 \mathrm{~Hz}$ (Type 5233A2, Kistler, AG Winterthur, Switzerland) during quiet standing. Then, percentage of the time for the deviation from the Center of Pressure (COP) toward one of the feet along mediolateral direction was calculated using MATLAB. These calculations were based on the relative position and deviation of COP with respect to the midpoint of the medial malleoli markers.

\section{Data analysis and statistical analysis}

The obtained kinematic and kinetic data were low-pass filtered, using a fourth-ordered Butterworth filter with zero-phase lag [23]. Force-plate signals were filtered with a cutoff frequency of $10 \mathrm{~Hz}$ [23]. Also, a cutoff frequency of $5 \mathrm{~Hz}$ was chosen for the kinematic data, based on residual analysis method [23]. The algebraic addition of time series data of angular accelerations of the lower limb joints and trunk segment throughout the quiet standing in the anteroposterior direction was calculated, and considered as the summation of their angular accelerations (Equation 1):

summation of angular accelerations of joints and trunks $=\sum_{i=1}^{n} a_{\text {ankle }}+a_{\text {knee }}+a_{\text {hip }}+a_{\text {trunk }}$ 


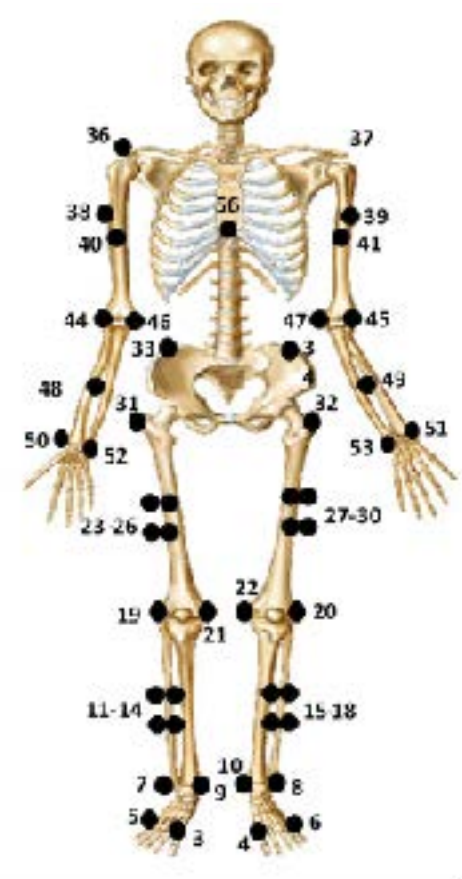

Figure 1. The marker positions in the experimental set-up

, where $\alpha$ denotes the joints and trunk angular accelerations. First, the angular displacement of the ankle, knee, hip, and trunk were calculated using rotation matrices of the segments. Then, the related angular accelerations were calculated by second time differentiation of the angular displacements in the anteroposterior direction [19]. Multiple linear regression model was performed to determine the most robust predictors of the COM acceleration. The dependent variable was COM acceleration. The angular accelerations of the joints and the summation of angular accelerations of joints and trunk served as the predictor variables. The regression analysis was performed in two separate parts. First, the accuracy of predicting COM acceleration was investigated based on angular acceleration of each lower limb joints and trunk. This investigation was carried out with enter regression, and if there was more than one appropriate predictor variable, stepwise regression method was applied for preparing the appropriate model. In the second part, predicting COM acceleration was done based on the summation of the angular accelerations of the lower limb joints and trunk, and enter regression was used in order to investigate the accuracy of this prediction. Finally, the obtained results were compared and the most accurate variable for predicting $\mathrm{COM}$ acceleration was determined. For presentation of the results, total variance was reported by the coefficient of determination, $\mathrm{R}^{2}$, and the respective level of significance. In addition, standardized coefficients (b coefficients) were also provided to indi-

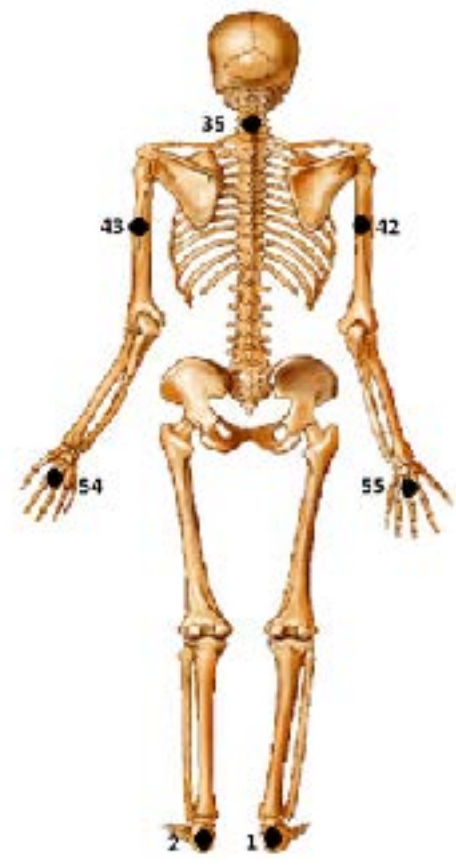

PHYSICAL TREA $\ M E N T S$

cate the relative importance of an independent variable on the dependent variable.

To compare the values of angular acceleration between different joints and the dominant and nondominant lower limbs, a univariate analysis of variance was conducted. Finally, paired $t$ test was performed to assess the significant difference in the weight distribution on dominant and nondominant lower limbs. All analyses were performed using SPSS (version 16.0) at a significance level of 0.05 .

\section{Results}

\section{Weight distribution}

The evaluations for percentage of time of COP deviation toward each foot, during quiet standing revealed that in $88 \%$ of trials ( 35 trials out of 40 ), the COP deviated toward the nondominant lower limb. In the remaining 5 trials, the deviation of COP toward the dominant and nondominant lower limbs was approximately the same during the tests (in $12 \%$ of trials). Figure 2 illustrates two patterns of COP displacement to lateral direction and its deviation toward the dominant and nondominant lower limbs. The negative and positive displacements indicated COP deviation towards the nondominant and dominant lower limbs during the tests, respectively. Paired $t$ test showed significant differences in COP deviation toward the lower limbs in all trials $(\mathrm{P}<0.001)$ in such a way that $\mathrm{COP}$ deviation was more toward nondominant leg. 
A

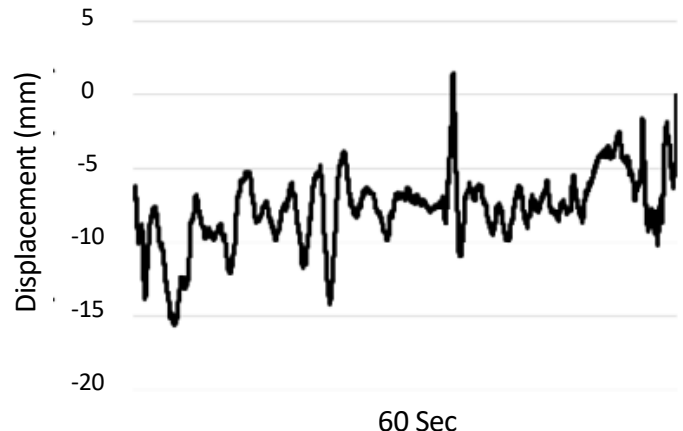

B

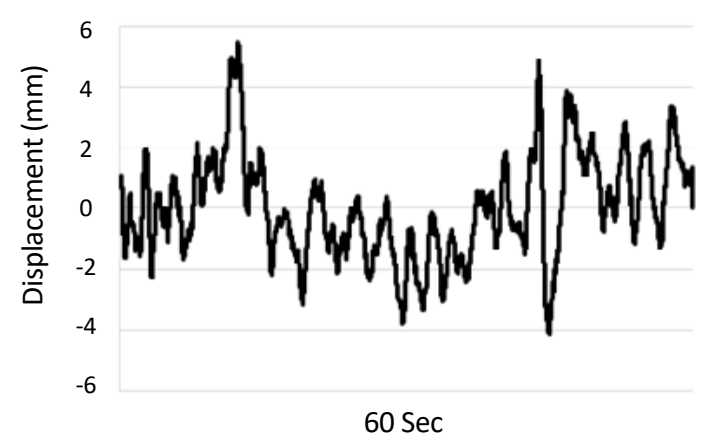

PHYSICAL TREATMENTS

Figure 2. COP displacement to lateral direction

The negative and positive displacements indicated COP deviation toward the nondominant and dominant lower limbs during the tests, respectively. (A): In $98 \%$ of test times, COP is deviated toward the nondominant lower limb (displacement values in $98 \%$ of test times are negative). (B): In $54 \%$ of test times, COP is deviated toward the nondominant lower limb (displacement values in $54 \%$ of test times are negative).

Therefore, it was revealed from the findings that subjects substantially supported their body weight on the nondominant lower limbs during quiet standing.

\section{Joints angular kinematics}

Judging by comparison of angular displacement of joints in lower limbs, the root mean square (RMS) values of angular displacement increased from distal joints to proximal (Figure 3A) ones. Based on the results, RMS values of angular displacement of hip were higher than knee and ankle in dominant and nondominant lower limbs, but this difference was only significant in ankle $(P<0.001)$. The results also revealed significantly higher RMS values of trunk angular displacement compared to RMS values of angular displacement of ankle and knee in both lower limbs, and hip in dominant lower limb $(\mathrm{P}<0.001)$. According to the analysis of variance, there was no significant difference between RMS values of angular displacement of joints in dominant and nondominant lower limbs. Based on these results, the RMS values of angular acceleration in lower limbs increased from distal joint to proximal ones $(\mathrm{P}<0.001)$. The greatest angular acceleration values were related to the hip (48.71 deg/ $\left./ \mathrm{s}^{2}\right)$, knee (31.86 deg/ $\left./ \mathrm{s}^{2}\right)$ and ankle (13.89 deg/ $\mathrm{s}^{2}$ ), respectively (Figure 3B). The RMS values related to trunk also revealed that trunk angular acceleration was not significantly different from that of hip. Furthermore, mean RMS angular acceleration values of nondominant

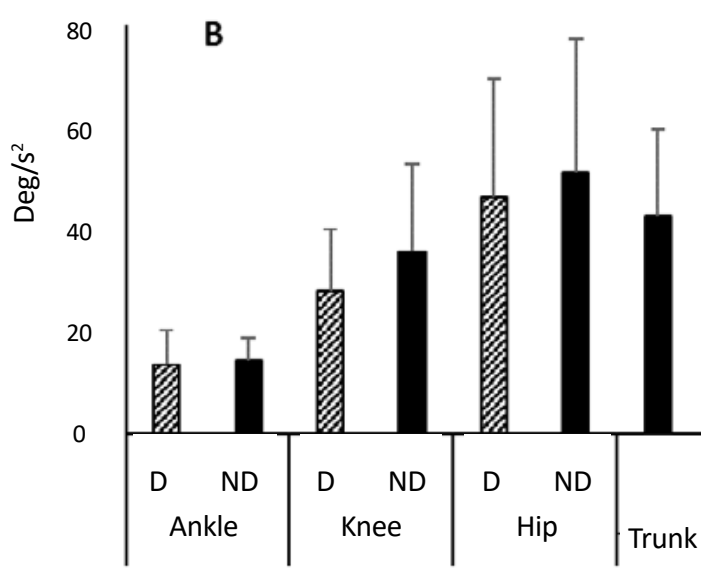

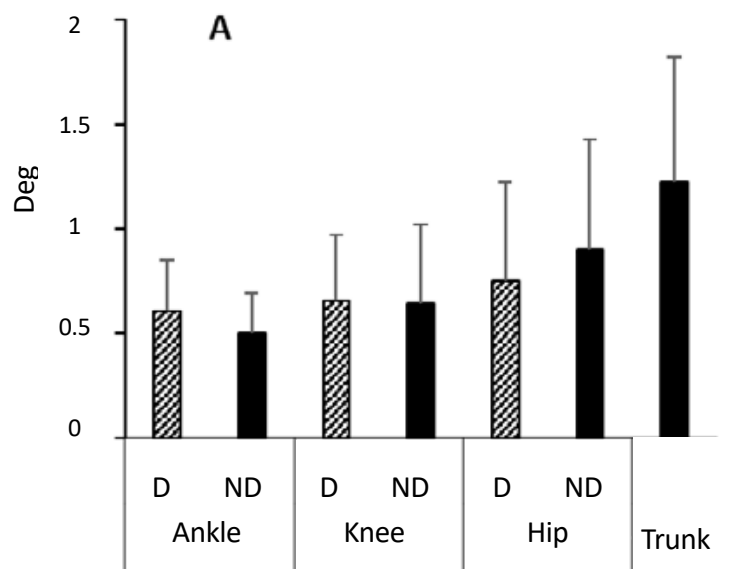

PHYSICAL TREA $\mid$ MENTS

Figure 3. Mean values of root mean square (RMS) of the angular displacement (A) and angular acceleration (B) at the joints of dominant (D) and nondominant (ND) lower limbs and trunk 
Table 2. The results of the regression analysis and model summary: statistical indicator of regression, analysis of variance, unstandardized and standardized coefficient

\begin{tabular}{|c|c|c|c|c|c|c|c|c|c|}
\hline \multicolumn{2}{|c|}{ Predictors } & $\mathbf{R}$ & $\mathbf{R}^{2}$ & Adjusted $\mathrm{R}^{2}$ & $\mathbf{F}$ & B & Beta & $\mathbf{t}$ & Sig. \\
\hline \multirow{3}{*}{ 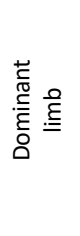 } & Ankle & 0.39 & 0.15 & 0.13 & 6.74 & 0.96 & 0.39 & 2.59 & $0.01 *$ \\
\hline & Knee & 0.07 & 0.01 & 0.02 & 0.2 & 0.1 & 0.07 & 0.44 & 0.66 \\
\hline & Hip & 0.65 & 0.42 & $0.4^{*}$ & 27.2 & 0.46 & 0.65 & 5.22 & $0.00^{*}$ \\
\hline \multirow{3}{*}{ 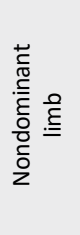 } & Ankle & 0.21 & 0.04 & 0.02 & 1.77 & 0.78 & 0.21 & 1.33 & 0.19 \\
\hline & Knee & 0.32 & 0.1 & 0.08 & 4.22 & 0.31 & 0.32 & 2.05 & 0.05 \\
\hline & Hip & 0.62 & 0.38 & $0.36^{*}$ & 23.22 & 0.39 & 0.62 & 4.82 & $0.00^{*}$ \\
\hline & Trunk & 0.19 & 0.04 & 0.01 & 1.42 & -0.19 & -0.19 & -1.19 & 0.24 \\
\hline
\end{tabular}

Predictors (RMS values of angular acceleration of the lower limbs joints and trunk); ${ }^{*} \mathrm{P}<0.01$

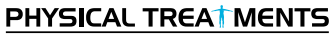

lower limb joints was higher than those of the dominant limb joints by $4.47 \mathrm{deg} / \mathrm{s}^{2}(\mathrm{P}<0.05)$.

\section{Prediction of COM acceleration based on the predictors}

Part 1: Predictors include angular acceleration of trunk and lower limb joints

As presented in Table 2, only hip angular acceleration in nondominant lower limb and ankle and hip angular acceleration in dominant lower limb can be appropriate variables for predicting $\mathrm{COM}$ acceleration $(\mathrm{P}<0.01)$. Thus, the effect of hip and ankle angular acceleration in dominant lower limb was studied with a stepwise method for predicting COM acceleration.
As a result of this analysis, the angular acceleration of ankle joint was excluded from the model and the final model was estimated based on the angular acceleration of the hip joint. Therefore, according to the results of this part, only the angular acceleration of hip joint was the appropriate variable for predicting COM acceleration in both dominant and nondominant lower limbs.

Part 2: Predictors include summation of angular accelerations of lower limb joints and trunk

The results of regression analysis in this part revealed that all four predictors introduced in Table 3 were appropriate variables for predicting $\mathrm{COM}$ acceleration

Table 3. The results of the regression analysis and model summary: statistical indicator of regression, analysis of variance, unstandardized and standardized coefficients

\begin{tabular}{|c|c|c|c|c|c|c|c|c|}
\hline Predictors & $\mathbf{R}$ & $\mathbf{R}^{2}$ & Adjusted $\mathrm{R}^{2}$ & $\mathbf{F}$ & B & Beta & $\mathbf{t}$ & Sig. \\
\hline Dominant joints & 0.74 & 0.54 & 0.53 & 45.18 & 0.56 & 0.74 & 6.72 & $0.00^{*}$ \\
\hline Nondominant joints & 0.72 & 0.52 & 0.51 & 41.23 & 0.54 & 0.72 & 6.42 & $0.00 *$ \\
\hline Dominant joints + Trunk & 0.96 & 0.92 & $0.91^{*}$ & 422.44 & 0.72 & 0.96 & 20.55 & $0.00 *$ \\
\hline Nondominant joints+trunk & 0.95 & 0.90 & $0.90^{*}$ & 355.54 & 0.71 & 0.95 & 18.86 & $0.00 *$ \\
\hline
\end{tabular}

Predictors (RMS values of the summation of angular accelerations of the lower limbs joints and trunk); $\mathrm{P}<0.01$ 
$(\mathrm{P}<0.001)$. Comparison of $\mathrm{R}^{2}$ adj values of the predictors (Table 3) showed that only a minimal difference exists between the accuracy of predicting COM acceleration based on summation of angular accelerations of dominant lower limb joints and nondominant ones. Moreover, when trunk angular acceleration is considered in calculating the summation, the accuracy of predicting COM acceleration increased. Accordingly, the amount of $\mathrm{R}_{\text {adj }}^{2}$ values related to the summation of angular accelerations of the dominant lower limb joints and trunk and nondominant ones attained 0.91 and 0.90 , respectively.

Overall, according to the results mentioned in parts 1 and 2 , as well as the comparison of $\mathrm{R}_{\text {adj }}^{2}$ values presented in tables 2 and 3, the summation of angular accelerations of trunk and dominant lower limb joints and nondominant ones were the most accurate predictor for COM acceleration.

The comparison of Mean Square Residual (MSR) values supported the results, too. As shown in Figure 4, MSR values of the summation of angular accelerations of trunk and joints in dominant and nondominant lower limbs were significantly lower than the MSR values of summation of angular accelerations of joints in dominant and nondominant lower limbs and MSR values of the hip accelerations in dominant and nondominant lower limbs.

\section{Discussion}

In this study, the accuracy of predicting COM acceleration by measuring the angular acceleration of trunk and joints in lower limbs was investigated in quiet standing. The results of weight distribution manner over the lower limbs demonstrated that although the body weight was transferred from one lower limb to another during quiet standing, in most cases the weight was transferred more to nondominant lower limb. Several studies revealed that individuals tend to distribute body weight unequally on legs during quiet standing [24, 25], and the lower-limb dominance role is an important issue [26]. Given that the joint kinematic variables of both dominant and nondominant legs were taken into consideration in this study, the proposed approach may provide more accurate results.

Comparing joint kinematics showed that the angular displacement and acceleration at lower limb joints and trunk increased from distal to proximal part of the body. Gage [27] study on angular displacement and Yamamato [13] on angular accelerations of leg joints confirmed the results of the present study. Therefore, in addition to the ankle, other lower limb joints and trunk have considerable movements and the body should be considered as a multi-segment system for COM prediction and balance assessment during quiet standing. In addition, although there was no difference between dominant and nondominant lower limbs in angular displacements of joints, the mean angular accelerations of joints was higher on nondominant lower limbs compared to the dominant ones. This finding was one of the important results of the present study, and to our knowledge it has never been reported in previous research studies.

Although, Günther et al. [11] compared the joints kinematic between left and right leg of the study subjects, no attention was paid to the dominant and nondominant legs, as well as the manner of weight distribution in their study. The reason for higher acceleration of joints in nondominant lower limbs compared to the dominant ones could be due to body weight distribution which is supported by nondominant or stability lower limb. Since angular acceleration is a more sensitive variable than angular displacement, the difference between joints performance in dominant and nondominant lower limbs during quiet standing has been recognized by angular acceleration.

The main questions of the present study were whether angular accelerations of lower limb joints and trunk was an appropriate variable in the prediction of COM acceleration, and whether accuracy of predicting COM acceleration based on joints angular accelerations was different between dominant and nondominant lower limb. The results showed that the summation of angular accelerations of joints was appropriate for predicting COM acceleration, because when COM acceleration was predicted from the angular acceleration of each joint separately, its accuracy was less than that of the summation of accelerations of all joints. The summation of angular accelerations of the joints, which is estimated based on Equation 1, indicates the coordinated or simultaneous changes of angular acceleration of the joints included in the equation. Thus, when the value of summation is low, increase in positive acceleration in one joint is coincided with increase in negative acceleration in another joint. Aramaki et al. [15] also observed the same pattern in changes of joint accelerations of hip and ankle joints and Yamamoto et al. [13] indicated that when accelerations of the ankle, knee and hip joints are considered together, the accuracy of predicting COM acceleration increases.

The novel point in the present study is considering the trunk acceleration in the calculation of the values of summation to predict COM acceleration, as well. Consequently, when COM acceleration is predicted based on the summation of accelerations of ankle, knee, hip, and trunk, the accuracy of the prediction will be increased, in comparison to when only the lower limb 


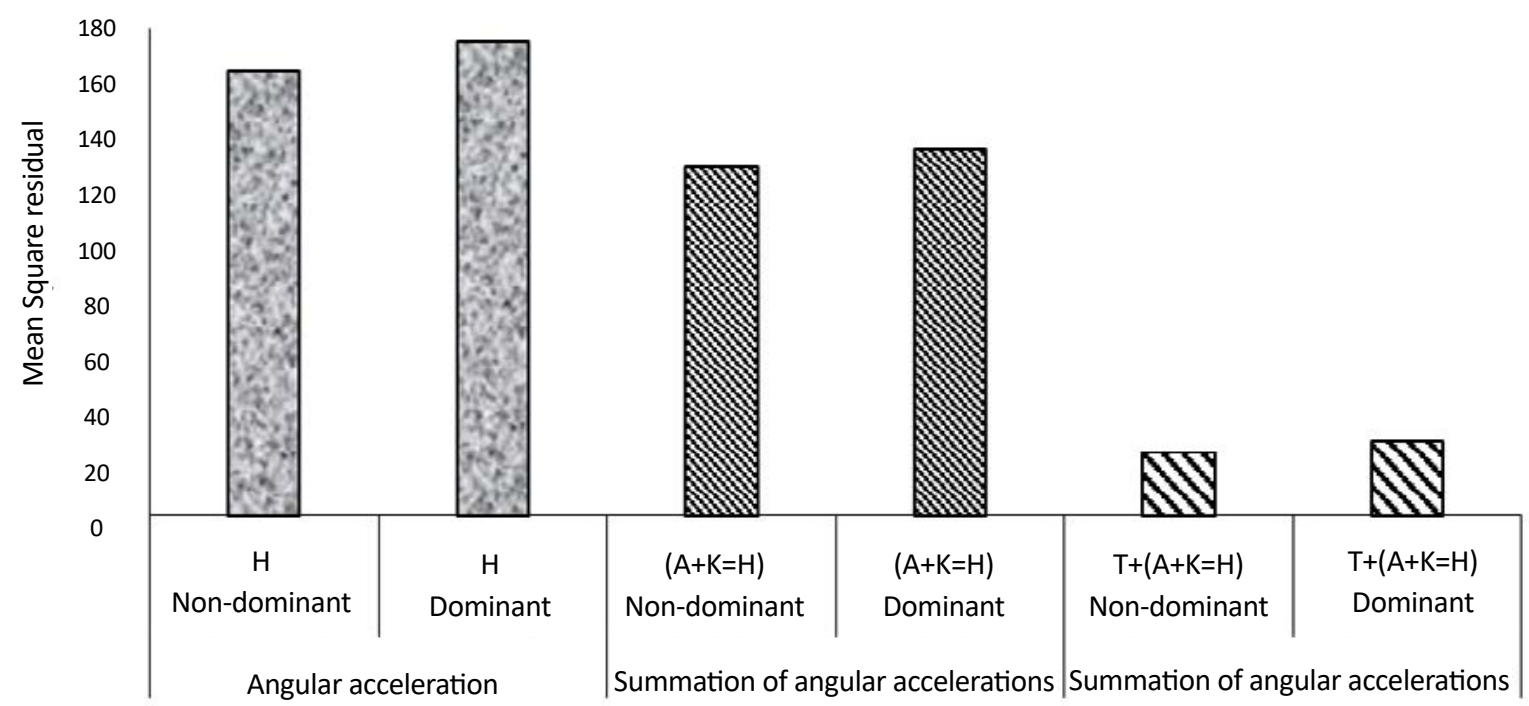

Figure 4. Mean square residual of the predictor variables with the highest adjusted $\mathrm{R}^{2}$

A, K, H and T denote the ankle, knee, hip joints and trunk, respectively.

joints are taken into account. In fact, simultaneous movement of trunk and lower limb joints resulted in better control of the COM. Hsu et al. [12], using uncontrolled manifold approach, revealed that during quiet standing balance control, the movement of joints and trunk was coordinated, but the coordinated pattern of joints was not specified in their study.

The present study revealed no significant difference between the accuracy of predicting COM acceleration based on the summation of accelerations of joints in dominant and nondominant lower limbs. However, the mean angular accelerations of the joints was higher at nondominant lower limbs compared to the dominant ones. Although the action of joints in the nondominant lower limbs was different from dominant ones due to weight bearing, their performance in each limb was coordinated in such a way that body COM be remained under control. Amanda et al. [26] reported that healthy individuals adopt different movement strategies to maintain balance during single-leg stance by nondominant limb compared to dominant one.

The results of the present study can obviate some ambiguities regarding balance control, especially in balance assessment. Although many studies have been carried out on balance assessment using different biomechanical variables, researchers are still seeking more appropriate criteria for assessing balance [7, 28]. Since it is believed that during postural control, the nervous system aims to control COM acceleration $[15,29]$ and the results of pres- ent study revealed that the summation of accelerations of lower limb joints and trunk can accurately predict COM acceleration, therefore, researchers can consider this variable for balance assessment. Furthermore based on strong biomechanical and physiological evidence, the joints acceleration is a factor in balance assessment [30, 31]. Physiological study of balance sensory systems also revealed the sensitivity of joint proprioceptors (which transfer the received information to the central nervous system) to joints velocity and acceleration. Therefore, the summation of angular accelerations of the lower limb joints and trunk is proposed as a valuable variable in assessing balance.

In clinical settings, where patients with neuromuscular abnormalities are under balance assessment, one important concern is to minimize the time required for the performance of experimental procedures. The proposed approach can predict the body COM acceleration based on only the lower limb joints and trunk accelerations. Therefore, it seems to be an appropriate alternative to the current time consuming anthropometric methods in assessing balance control in impaired individuals.

It should be noted that using Visual 3D together with Qualisys motion capture system (as a biomechanical modeling tool for data analyses and the method derived from Dempster for body segment inertia parameter estimates) could be regarded as limitations of this study, with regard to generalizing the findings to other kinematic models and obtaining body segment parameters. 
Therefore, other methods and models should be used that allow a comparison of any potential variation in the accuracy of similar data for better description of future investigations.

In conclusion, the summation of angular accelerations of the trunk segment and joints in lower limbs is an appropriate variable for predicting COM acceleration. The accuracy of this prediction by using the accelerations of joints was the same in dominant and nondominant lower limbs. It is recommended that researchers consider body as a multi-segment system and attend to the performance of the trunk along with the lower limb joints during quiet standing balance assessment.

\section{Acknowledgements}

This research did not receive any specific grant from funding agencies in the public, commercial, or notfor-profit sectors. The authors would like to thank Dr. Mohammadtaghi Karimi and the staff of Musculoskeletal Research Center affiliated to Isfahan University of Medical Sciences, Isfahan, Iran, for their support during data collection.

\section{Conflict of Interest}

The authors declared no conflicts of interest.

\section{References}

[1] Peterka RJ. Sensorimotor Integration in Human Postural Control. Journal of Neurophysiology. 2002; 88(3):1097-118. doi: $10.1152 /$ jn.2002.88.3.1097

[2] Massion J. Movement, posture and equilibrium: Interaction and coordination. Progress in Neurobiology. 1992; 38(1):3556. doi: 10.1016/0301-0082(92)90034-c

[3] Hasan SS, Robin DW, Shiavi RG. Drugs and postural sway: quantifying balance as a tool to measure drug effects. IEEE Engineering in Medicine and Biology Magazine. 1992 Dec;11(4):35-41. doi: 10.1109/51.256956

[4] Ji Z, Findley T, Chaudhry H, Bukiet B. Computational method to evaluate ankle postural stiffness with ground reaction forces. The Journal of Rehabilitation Research and Development. Journal of Rehabilitation Research \& Development; 2004; 41(2):207. doi: 10.1682/jrrd.2004.02.0207

[5] Barbier F, Allard P, Guelton K, Colobert B, Godillon-Maquinghen AP. Estimation of the 3-d center of mass excursion from force-plate data during standing. IEEE Transactions on Neural Systems and Rehabilitation Engineering. 2003; 11(1):31-7. doi: 10.1109/tnsre.2003.810433
[6] Chaudhry H, Bukiet B, Ji Z, Findley T. Measurement of balance in computer posturography: Comparison of methods - A brief review. Journal of Bodywork and Movement Therapies. 2011; 15(1):82-91. doi: 10.1016/j.jbmt.2008.03.003

[7] Ruhe A, Fejer R, Walker B. The test-retest reliability of centre of pressure measures in bipedal static task conditions - A systematic review of the literature. Gait \& Posture. 2010; 32(4):436-45. doi: 10.1016/j.gaitpost.2010.09.012

[8] Morasso PG, Spada G, Capra R. Computing the COM from the $\mathrm{COP}$ in postural sway movements. Human Movement Science. 1999; 18(6):759-67. doi: 10.1016/s01679457(99)00039-1

[9] Winter D. Human balance and posture control during standing and walking. Gait \& Posture. 1995; 3(4):193-214. doi: 10.1016/0966-6362(96)82849-9

[10] Kobravi H-R, Erfanian A. A decentralized adaptive fuzzy robust strategy for control of upright standing posture in paraplegia using functional electrical stimulation. Medical Engineering \& Physics. 2012; 34(1):28-37. doi: 10.1016/j.medengphy.2011.06.013

[11] Günther M, Grimmer S, Siebert T, Blickhan R. All leg joints contribute to quiet human stance: A mechanical analysis. Journal of Biomechanics. 2009; 42(16):2739-46. doi: 10.1016/j. jbiomech.2009.08.014

[12] Hsu W-L, Scholz JP, Schöner G, Jeka JJ, Kiemel T. Control and Estimation of Posture During Quiet Stance Depends on Multijoint Coordination. Journal of Neurophysiology. 2007; 97(4):3024-35. doi: 10.1152/jn.01142.2006

[13] Yamamoto A, Sasagawa S, Oba N, Nakazawa K. Behaviora effect of knee joint motion on body's center of mass during human quiet standing. Gait \& Posture. 2015; 41(1):291-294. doi: 10.1016/j.gaitpost.2014.08.016

[14] Zhang H, Nussbaum MA, Agnew MJ. Development of a sliding mode control model for quiet upright stance. Medical Engineering \& Physics. 2016; 38(2):204-8. doi: 10.1016/j. medengphy.2015.11.019

[15] Aramaki Y, Nozaki D, Masani K, Sato T, Nakazawa K, Yano H. Reciprocal angular acceleration of the ankle and hip joints during quiet standing in humans. Experimental Brain Research. 2001; 136(4):463-73. doi: 10.1007/s002210000603

[16] Sasagawa S, Ushiyama J, Kouzaki M, Kanehisa H. Effect of the hip motion on the body kinematics in the sagittal plane during human quiet standing. Neuroscience Letters. 2009; 450(1):27-31. doi: 10.1016/j.neulet.2008.11.027

[17] Scholz JP, Schöner G, Hsu WL, Jeka JJ, Horak F, Martin V. Motor equivalent control of the center of mass in response to support surface perturbations. Experimental Brain Research. 2007; 180(1):163-79. doi: 10.1007/s00221-006-0848-1

[18] Taheri AR, Karimi MT. Evaluation of the gait performance of above-knee amputees while walking with 3R20 and 3R15 knee joints. Journal of Research in Medical Sciences. 2012; 17(3):258-63. PMCID: PMC3527044

[19] Dempster WT. Space requirements of the seated operator (technical report). New York: Wright-Patterson Air Force Base Ohio. 1995.

[20] Hoffman M, Schrader J, Applegate T, Koceja D. Unilateral postural control of the functionally dominant and non- 
dominant extremities of healthy subjects. Journal of Athletic Training. 1998; 33(4):319-322. PMCID: PMC1320581

[21] Velatto J, Weyer J, Ramirez A, Winstead J, Bahamonde R. Relationship between leg dominance tests and type of task. Journal of Sports Sciences. 2011; 11(2):1035-1038.

[22] Sadeghi H, Allard P, Prince F, Labelle H. Symmetry and limb dominance in able-bodied gait: a review. Gait \& Posture. 2000; 12(1):34-45. doi: 10.1016/s0966-6362(00)00070-9

[23] Winter D. Biomechanics and motor control of human movement. $3^{\text {rd }}$ edition. New York: Wiley; 2004.

[24] Rougier PR. Relative contribution of the pressure variations under the feet and body weight distribution over both legs in the control of upright stance. Journal of Biomechanics. 2007; 40(11):2477-82. doi: 10.1016/j.jbiomech.2006.11.003

[25] Genthon N, Rougier P. Influence of an asymmetrical body weight distribution on the control of undisturbed upright stance. Journal of Biomechanics. 2005; 38(10):2037-49. doi: 10.1016/j.jbiomech.2004.09.024

[26] Clifford AM, Holder-Powell H. Postural control in healthy individuals. Clinical Biomechanics. 2010; 25(6):546-51. doi: 10.1016/j.clinbiomech.2010.03.005

[27] Gage WH, Winter DA, Frank JS, Adkin AL. Kinematic and kinetic validity of the inverted pendulum model in quiet standing. Gait \& Posture. 2004; 19(2):124-32. doi: 10.1016/ s0966-6362(03)00037-7

[28] Cavanaugh JT, Guskiewicz KM, Stergiou N. A Nonlinear Dynamic Approach for Evaluating Postural Control. Sports Medicine. 2005; 35(11):935-50. doi: 10.2165/00007256200535110-00002

[29] Gurfinkel VS, Ivanenko YP, Levik YS, Babakova IA. Kinesthetic reference for human orthograde posture. Neuroscience. 1995; 68(1):229-43. doi: 10.1016/0306-4522(95)00136-7

[30] Rougier P-R. What insights can be gained when analysing the resultant centre of pressure trajectory? Neurophysiologie Clinique/Clinical Neurophysiology. 2008; 38(6):363-73. doi: 10.1016/j.neucli.2008.09.006

[31] Woodhull AM, Maltrud K, Mello BL. Alignment of the human body in standing. European Journal of Applied Physiology and Occupational Physiology. 1985; 54(1):109-15. doi: $10.1007 /$ bf00426309 